\title{
Methacrylic Functionalized Hybrid Carbon Nanomaterial for the Selective Adsorption and Detection of Progesterone in Wastewater
}

Xia Cui

Xi'an Jiaotong University

Hua Shu

Xi'an Jiaotong University

Lu Wang

Xi'an Jiaotong University

Guoning Chen

Xi'an Jiaotong University

Jili Han

Xi'an Jiaotong University

Qianqian Hu

Xi'an Jiaotong University

Kamran Bashir

Xi'an Jiaotong University

Zhimin Luo

Xi'an Jiaotong University

Chun Chang

Xi'an Jiaotong University

Jia Zhang

Shannxi Hangjiang Pharmaceutical Group Co., Ltd

Qiang Fu ( $\square$ fuqiang@mail.xjtu.edu.cn )

Xi'an Jiaotong University School of Medicine

\section{Research Article}

Keywords: Progesterone, Methacrylic, Hybrid carbon nanomaterial, Solid phase extraction, Endocrinedisrupting chemical, Wastewater

Posted Date: March 25th, 2021

DOl: https://doi.org/10.21203/rs.3.rs-330364/v1 
License: (c) (i) This work is licensed under a Creative Commons Attribution 4.0 International License. Read Full License

Version of Record: A version of this preprint was published at Environmental Science and Pollution Research on June 30th, 2021. See the published version at https://doi.org/10.1007/s11356-021-15056-1. 

6

\section{8}

Methacrylic functionalized hybrid carbon nanomaterial for the selective adsorption and detection of progesterone in wastewater 


\section{Abstract}

Progesterone, an endocrine-disrupting chemical, has been frequently detected in wastewater for decades, posing a serious threat to ecological and human health. However, it is still a challenge to achieve the effective detection of progesterone in complex matrices water samples. In this study, a novel adsorbent CNT@CS/P(MAA) was prepared by grafting methacrylic polymers on the surface of modified carbon nanomaterials. Compared with other reported materials, the hybrid carbon nanomaterial could selectively identify the progesterone in the complex industrial pharmaceutical wastewater, and its adsorption performance is almost independent of $\mathrm{pH}$ and environmental temperature. In addition, this nanomaterial could be reused with a good recovery rate. The prepared nanomaterials were characterized by transmission electron microscopy, Fourier transform infrared spectroscopy, X-ray diffraction, nitrogen adsorption and desorption experiments and thermo gravimetric analysis. The results confirmed that the methacrylic polymers and chitosan layer were successfully grafted on the surface of carbon nanotubes. Adsorption isotherms, adsorption kinetics, and selectivity tests showed that CNT@CS/P(MAA) had a high adsorption capacity (44.45 mg. $\mathrm{g}^{-1}$ ), a fast adsorption rate and a satisfied selectivity for progesterone. Then, CNT@CS/P(MAA) was used as solid phase extraction sorbent and combined with HPLC to enrich progesterone from the wastewater samples. Under the optimum conditions, a good linearity was obtained with the correlation coefficient was 0.9998 , and the limit of detection was $0.003 \mathrm{ng} \cdot \mathrm{mL}^{-1}$. Therefore, this method could be used for the selective and effective detection of progesterone in the industrial wastewater with complex substrates, and provided a new method for the detection of progesterone in other environmental waters.

\section{Keywords}

Progesterone; Methacrylic; Hybrid carbon nanomaterial; Solid phase extraction; Endocrine-disrupting chemical; Wastewater. 


\section{Highlights}

41 1. A methacrylic functionalized hybrid carbon nanomaterial was synthesized.

42 2. The prepared nanocomposites could selectively recognize and enrich P4 in water.

43 3. The adsorption capacity of this material for $\mathrm{P} 4$ is $44.45 \mathrm{mg} \cdot \mathrm{g}^{-1}$.

44 4. The adsorption performance of $\mathrm{P} 4$ is hardly affected by $\mathrm{pH}$ and adsorption temperature.

45 5. The SPE-HPLC method could be used to detect P4 in real industrial wastewater.

46 


\section{Introduction}

The presence of endocrine-disrupting chemicals (EDCs) in the environment has attracted extensive attention from the public for their ability to impair reproductive and immune functions of aquatic organisms (Du et al. 2018, Zhang et al. 2017). These chemicals include natural and synthetic hormones as well as their metabolites (Hashmi et al. 2020). Previous researches mostly focused on estrogen and androgens(Luque-Cordoba et al. 2020, Tian et al. 2020, Zhang et al. 2020b). However, there is growing awareness that other EDCs in the environment, such as progesterone ( $\mathrm{P} 4$,$) , may also affect the health of$ aquatic organisms and human (Fabbrocini et al. 2019, Hashmi et al. 2018).

Progesterone (Fig. 1), a C-21 structural steroid hormone, can help to modulate the menstrual cycle, making the uterus ready for pregnancy, and affect the breast development and lactation (Graham $\&$ Clarke 1997). It has been widely applied in breast cancer therapy, endocrine regulation, drug contraception and assisted reproduction (De Lima \&Spinelli 2013, Guohua et al. 2017, Kumar et al. 2015). However, it has become a main contaminant in the aquatic environment due to the massive discharge of industrial waste and urban sewage (Hashmi et al. 2020). In China, the production and application of $\mathrm{P} 4$ is huge, due to the front-end advantage of natural resources of diosgenin plants such as Dioscorea zingiberensis (Guo et al. 2016). At the same time, P4 can be used as a synthetic precursor of other steroid hormones, which also increases the production of P4. Once a large amount of P4 remaining in industrial production wastewater is discharged into the environment, it will pose a great threat to animal and human health. (Kumar et al. 2015, Yu et al. 2019). However, it is still a challenge to effectively detect and enrich the residual P4 in the industrial wastewater due to the complex substrate, many interfering coexisting substances and wide range of $\mathrm{pH}$ values. Therefore, developing an effective method to monitor P4 in the industrial waste is necessary. Considering the concentration of P4 in the industrial water, such methods not only suit for the complex media, but also require high sensitivity.

To date, several analytical methods such as thin layer chromatography (TLC) (Chamas et al. 2017), gas chromatography (GC) (Nezhadali et al. 2016), high performance liquid chromatography (HPLC) (Kollofrath et al. 2020), capillary electrophoresis (Siren \&El Fellah 2017), gas chromatography-mass spectrometry (GC/MS) (Siren \&El Fellah 2017), liquid chromatography-mass spectrometry (LC-MS) (Zong et al. 2018), electrochemical sensor (Akshaya et al. 2019), and enzyme linked immunosorbent assay (ELISA) tests (Zhang et al. 2020c) have been used to determine the presence of $\mathrm{P} 4$ in environmental samples, especially in the wastewater. HPLC is the most frequently used method because of the low cost, high performance, relatively good sensitivity and stability. Roya Mirzajani et al. (Mirzajani et al. 2019a) reported a nanocomposite consisiting of graphene oxide, ZIF-8 metal-organic frameworks and molecularly imprinted polymers, which was used as the sorbent of solid phase microextraction coupled with HPLC to recognize and separate five sterol and steroid hormones in biological samples. This method could detect five sterol and steroid hormones simultaneously and had a low detection limit (3-5 ng. $\left.\mathrm{L}^{-1}\right)$. However, it is limited by the $\mathrm{pH}$ of the samples, and had some problems such as low adsorption capacity and potential template leakage. Kasre Razmkhah et al. (Khan et al. 2019) synthesized a magnetic carbon nanotube-based strontium-titanium ( $\left.\mathrm{Fe} / \mathrm{CNT}-\mathrm{SrTiO}_{3}\right)$ adsorbent, which could extract 17ß-estradiol, ethinyl estradiol and progesterone from milk coupled with MSPE-HPLC. This method had good stability but poor specificity. In order to improve the specificity of analytical methods, biological molecules such 
as antibodies (Guo et al. 2019), enzymes (Xu et al. 2020) and DNA aptamers (Tao et al. 2020) were used as recognition tools. Khan Muhammad et al.(Khan et al. 2019) devised an ultra-sensitive electrochemical-digital sensor chip for monitoring cortisone in real time with anti-cortisol antibody as recognition elements. Xu Xuan et al. (Xu et al. 2018) fabricated an electrochemical enzymatic nanoreactor by immobilizing CYP3A4 inside polydopamine modified nanoporous graphene foams, which was successfully applied to detect three steroid hormones. Those methods had achieved superior selectivity, but often suffer from unstable, easily deactivated and high price, and cannot be applied to complex industrial wastewater. Thus, it is urgent to develop a novel recognition material for industrial wastewater with simple, stable, excellent adsorption performance and high specificity.

Nowadays, carbon nanotubes (CNTs) have received much attention due to their outstanding properties, such as narrow pore distribution, large $\left(100\right.$ to $\left.\geq 500 \mathrm{~m}^{2} \cdot \mathrm{g}^{-1}\right)$ specific surface area, and excellent thermal and mechanical resistance (Shu et al. 2020, Xu et al. 2017). However, the use of CNTs is limited for their easy agglomeration, hydrophobicity and biological toxicity (Jakubus et al. 2016). Studies have shown that the surface-carrying groups of CNTs can be changed by surface modification to improve their properties (Chen et al. 2018, Liu et al. 2018). In our previous study, we reported a method of modifying carbon nano-material with chitosan. The prepared carbon micrcoil-chitasan composites showed good solubility, dispersibility and pH-controllability (Hua et al. 2018).

Herein, we intend to fabricate a selective sorbent by grafting the methacrylic acid polymer onto the surface of carbon nanotubes modified with chitosan. The prepared material was served as the adsorbent of solid phase extraction column and combined with high-performance liquid chromatography (HPLC) for the selective enrichment and determination of P4. The hybrid carbon nanomaterial modified with methacrylic acid polymers (CNT@CS/P(MAA) was analyzed by transmission electron microscopy (TEM), Fourier transform infrared spectroscopy (FT-IR), X-ray diffraction (XRD), nitrogen adsorption and desorption experiments and thermo gravimetric analysis (TGA). The adsorption isotherms and kinetics were studied to elucidate the adsorption mechanism. Moreover, the $\mathrm{pH}$ value, temperature and other parameters affecting adsorption efficiency and recovery were also investigated. Finally, the established SPE-HPLC method was applied to detect P4 in the real industrial wastewater samples.

\section{Experiment}

\subsection{Reagents and chemicals}

Progesterone, estrone (E) and estradio (E2) were purchased from Wuhan Dong Kangyuan Technology (Hubei, China). Dexamethasone (DXM), bisphenol A (BPA) and diethylstilbestrol (DES) were provided by Hubei Kang Baotai Fine Chemical (Hubei, China). Methacrylic acid (MAA) was provided by Tianjin Chemical Reagent Plant (Tianjin, China) and distilled under vacuum to remove inhibitors before use. Ethylene glycol dimethacrylate (EGDMA) was obtained from Sigma-Aldrich (New Jersey, USA) and refined by distillation. 2,2'-azobisisobutyronitrile (AIBN) was purchased from Shanghai No.4 Reagent Factory (Shanghai, China) and recrystallized from methanol before use. Chitosan $\left(\mathrm{MW}=600000 \mathrm{~g} \cdot \mathrm{mol}^{-1}\right)$ with a $90 \%$ degree of deacetylation was obtained from Sinopharm Chemical Reagent Co., Ltd (Shanghai, China). Glutaraldehyde (25\% aqueous solution) was purchased from Tianjin Fuchen Chemical Reagents Factory (Tianjin, China). HPLC-grade methanol was purchased from Tianjin Kemiou Chemical Reagent Co. (Tianjin, China). Ultrapure water was prepared using a Molement $1805 \mathrm{~b}$ 
purification system (Shanghai, China). And all other reagents were of analytical grade. Progesterone standard solutions were diluted with $1.0 \mathrm{mg} \cdot \mathrm{mL}^{-1}$ progesterone stock solution and prepared temporarily before use. The real industrial wastewater samples were collected from a local pharmaceutical enterprise in Hanzhong, Shannxi.

\subsection{HPLC conditions}

A Shimadzu high performance liquid chromatograph system (LC 2010A HT, Kyoto, Japan) with an ultraviolet detector was employed for the determination of target. The analytical column $(4.6 \times 250 \mathrm{~mm})$ was a $5 \mu \mathrm{m} \mathrm{C18} \mathrm{column.} \mathrm{The} 90 \%$ methanol solution was used as the mobile phase with a flow rate of 1 $\mathrm{mL} \min ^{-1}$. The detection wavelength was $254 \mathrm{~nm}$ and the column temperature was kept at $30^{\circ} \mathrm{C}$.

\subsection{Preparation of CNT@CS/P(MAA)}

CNT@CS complex was synthesized according to our previous report (Liu et al. 2017) with slight modification, which was described in supplementary material. The fabrication of CNT@CS/P(MAA) was shown below: $500 \mathrm{mg}$ of CNT@CS was mixed with $100 \mathrm{~mL}$ of methanol. Subsequently, the MAA, EGDMA, and initiator AIBN were added in sequence. After 5 minutes of ultrasound, the reaction was performed in a shaker at $50{ }^{\circ} \mathrm{C}$ for 6 hours. The obtained products were rinsed several times with purified water and methanol to remove the unreacted substances and impurities, and dried at $50{ }^{\circ} \mathrm{C}$ overnight. The resulting compounds were the CNT@CS/P(MAA).

\subsection{Characterization of CNT@CS /P(MAA)}

TEM images were recorded on a JEM2100 transmission electron microscopy (TEM, JEOL Co., Japan). FT-IR spectra were performed on a Nicolet iS10 spectrometer (Thermo Fisher Scientific, America) in the range of 400 to $4000 \mathrm{~cm}^{-1}$. The crystalline structures of the obtained materials were characterized by XRD (Shimadzu, Japan). The surface area of the polymers was measured by nitrogen adsorption and desorption experiments using an Autochem 2920 physical chemistry analyzer (Quantachrome, USA). Brunauer-Emmett-Teller (BET) theory and the Barrett-Joyner-Halenda (BJH) theory were used to calculate the specific surface area and the average pore diameter. TGA was operated on an SDT Q600 thermogravimetric analyzer (New Castle, USA) with a ramp of $10^{\circ} \mathrm{C} \cdot \mathrm{min}^{-1}$ from room temperature to $800{ }^{\circ} \mathrm{C}$.

\subsection{Adsorption test}

2.5.1 Optimization of adsorption conditions

In order to explore the optimum conditions of adsorption experiments, the water contents, $\mathrm{pH}$ and adsorption temperature were investigated.

The water contents were optimized by following steps: $\mathrm{P} 4$ stock solution $\left(1 \mathrm{mg} \cdot \mathrm{mL}^{-1}\right)$ was prepared with methanol and diluted to $50 \mu \mathrm{g} \cdot \mathrm{mL}^{-1}$ with various methanol-water solutions. The water content of the working solutions was ranged from $10 \%$ to $80 \%$ due to the solubility of progesterone. Subsequently, $2 \mathrm{~mL}$ of $\mathrm{P} 4$ working solution was mixed with $5 \mathrm{mg}$ of adsorbent and oscillated 90 minutes at $25^{\circ} \mathrm{C}$. After filtration, the concentration of $\mathrm{P} 4$ in the supernatant was detected by HPLC. And the adsorption capacity $Q\left(\mathrm{mg} \cdot \mathrm{g}^{-1}\right)$ was calculated according to the following formula:

$$
Q=\left(C_{0}-C_{\mathrm{f}}\right) \times V / \mathrm{m}
$$


where $Q\left(\mathrm{mg} \cdot \mathrm{g}^{-1}\right)$ was the adsorption capacity of the sorbent for $\mathrm{P} 4 ; C_{0}\left(\mathrm{mg} \cdot \mathrm{mL}^{-1}\right)$ and $C_{\mathrm{f}}\left(\mathrm{mg} \cdot \mathrm{mL}^{-1}\right)$ were the initial and final concentration of $\mathrm{P} 4$ in the working solution, respectively. $V(\mathrm{~mL})$ was the volume of the working solution, and $\mathrm{m}(\mathrm{g})$ was the weight of the sorbent.

The $\mathrm{pH}$ and adsorption temperature were optimized by the same method as above, except that the $\mathrm{pH}$ value of the aqueous phase (adjusted to 3.0, 5.0, 7.0, 9.0, 11.0 by adding $\mathrm{HCl}$ or $\mathrm{NaOH}$ ) and the adsorption temperature $\left(25^{\circ} \mathrm{C}, 35^{\circ} \mathrm{C}, 45^{\circ} \mathrm{C}\right)$ were changed.

\subsubsection{Adsorption isotherms}

Under the optimum conditions, isothermal adsorption experiments were investigated to explore the adsorption property and principle of the adsorbent to the analyte. The adsorbent was mixed with various P4 standard solutions of different concentrations $\left(5,15,25,50,75,100,125,150\right.$ and $\left.175 \mu \mathrm{g} \cdot \mathrm{mL}^{-1}\right)$. After reacting 120 minutes at $25^{\circ} \mathrm{C}, 35^{\circ} \mathrm{C}$ and $45^{\circ} \mathrm{C}$, the supernatants were filtered and determined by HPLC. The adsorption capacity of the sorbent for P4 at different concentrations was calculated by Eq. (2),

$$
Q_{e}=\left(C_{0}-C_{e}\right) \times V / \mathrm{m}
$$

where $Q_{e}\left(\mathrm{mg} \cdot \mathrm{g}^{-1}\right)$ was the adsorption capacity of the sorbent for P4 at different concentrations; $C_{0}$ $\left(\mathrm{mg} \cdot \mathrm{mL}^{-1}\right)$ represented the initial concentration of $\mathrm{P} 4$ in the working solution; $C_{e}\left(\mathrm{mg} \cdot \mathrm{mL}^{-1}\right)$ was the final concentration of $\mathrm{P} 4$ in different solution; $V(\mathrm{~mL})$ was the volume of the working solution, and $\mathrm{m}(\mathrm{g})$ was the weight of the sorbent.

\subsubsection{Adsorption kinetics}

To study the adsorption process and determine the equilibrium time, an adsorption kinetics experiment was carried out. The specific operation was consistent with the description in the section of 2.5.2, except that the adsorption time was changed to $0.17,1,5,10,30,60,120$ and 150 minutes. The adsorption capacity of the sorbent for $\mathrm{P} 4$ at different time was calculated by Eq. (3),

$$
Q_{t}=\left(C_{0}-C_{t}\right) \times V / \mathrm{m}
$$

where $Q_{t}\left(\mathrm{mg} \cdot \mathrm{g}^{-1}\right)$ was the adsorption capacity of the sorbent for P4 at different time, $C_{0}\left(\mathrm{mg} \cdot \mathrm{mL}^{-1}\right)$ represented the initial concentration of $\mathrm{P} 4$ in the solution, $C_{\mathrm{t}}\left(\mathrm{mg} \cdot \mathrm{mL}^{-1}\right)$ was the $\mathrm{P} 4$ concentration in the solution at time t, $V(\mathrm{~mL})$ was the volume of the working solution, and $\mathrm{m}(\mathrm{g})$ was the weight of the sorbent.

\subsubsection{Adsorption selectivity}

E, E2, DXM, DES and BPA (Fig. 1) were chosen to investigate the selectivity performance of the CNT@CS/P(MAA). The selectivity of the adsorbent was measured by the selection coefficient $S C_{P 4 / R}$, which was calculated by Eq. (4),

$$
S C_{P 4 / R}=Q_{P 4} / Q_{R}
$$

where $Q_{P 4}\left(\mathrm{mg} \cdot \mathrm{g}^{-1}\right)$ was the adsorption capacity of the adsorbent for $\mathrm{P} 4$, and $Q_{R}\left(\mathrm{mg} \cdot \mathrm{g}^{-1}\right)$ was the adsorption capacity for other reference compounds.

\subsection{SPE procedure}

A total of 10 mg CNT@CS/P(MAA) was packed in an empty SPE cartridge under room temperature. After being activated and rinsed by $2 \mathrm{~mL}$ of methanol and water successively, the sample solutions were slowly loaded onto the SPE column. Subsequently, the SPE column was washed with water to remove 
the water-soluble substances, then the bound targets were eluted with eluting solution. Finally, the eluents were evaporated with a stream of air. The residues were redissolved in methanol and analyzed by HPLC.

\subsection{Method validation and real samples analysis}

According to the recommendations of the International Conference on Harmonization Q2(R1), the developed SPE-HPLC system was validated with specificity, linearity, range, limit of detection (LOD), limit of quantification (LOQ), accuracy and precision.

In order to investigate the practicability of the method system, a batch of industrial wastewater samples were collected from the wastewater treatment system of a steroid hormone pharmaceutical company in Hanzhong, Shaanxi Province. No. 1 samples were collected in an anaerobic bacteria treatment tank closed to the industrial production, and No. 2 samples were collected at the end of the sewage treatment system, which would be discharged directly into the environment. After centrifuged 5 minutes by 4000rpm, those samples were applied to the method system.

\section{Results and discussion}

\subsection{Preparation of CNT@CS/P(MAA)}

The preparation process of CNT@CS/P(MAA) is shown in Fig. 2. CNT@CS was prepared by coating chitosan on the surface of CNTs through glutaraldehyde cross-linking. This method could effectively improve the water-solubility and dispersity of CNTs by introducing hydroxyl and amino groups on the chitosan layer. During the pre-polymerization process, the carboxyl groups of methacrylic acid were attracted by the amino groups on the surface of CNT@CS, so the methacrylic acid molecules were deposited on the carrier surface. Under the heat initiation, MAA polymers with porous structures were grafted on the surface of CNT@CS through free radical reaction. The CNT@CS/P(MAA) composites could be combined with P4 through electrostatic interaction (such as hydrogen bonds) and spatial interaction force. During the polymerization, the amount of MAA and EGDMA could affect the effective binding sites and the pore size of CNT@CS/P(MAA). Subsequently, the amount of MAA and EGDMA were optimized.

\subsubsection{The optimization of MAA}

In order to explore the optimal adsorption performance of CNT@CS/P(MAA), the amount of MAA was studied. As shown in Table S1, the results of CNT@CS/P(MAA) exhibit the highest adsorption capacity, when the mass ratio of carrier/MAA is 4:1. This is because when the amount of MAA is insufficient, sufficient binding sites cannot be provided. On the contrary, when the amount of MAA is too much, the binding sites will form competitive adsorption, which will also reduce the adsorption performance.

\subsubsection{The optimization of EGDMA}

The amount of EGDMA was important to control the pore size of the polymers, as well as the performance of the functional composites. Adsorption performance of the polymers with different amount of EGDMA are investigated, as shown in the Table S2. As the increases of the amount of EGDMA, the adsorption capacity of the material decreases gradually, while the selectivity performance shows the opposite phenomenon. When the amount of EGDMA increases, the degree of crosslinking of the polymers increases. As a result, the structure of the polymers becomes more denser and the cavities carried are smaller, which makes it more difficult for the analytes to enter and exit the pores freely. 
Therefore, the adsorption property of the polymers to $\mathrm{P} 4$ is reduced. However, the functional groups and three-dimensional structure of $\mathrm{P} 4$ analogues are different from $\mathrm{P} 4$. When the polymers structure becomes denser, it is more difficult for $\mathrm{P} 4$ analogues to pass through the pores and bind to the sites of action. Thus, the selectivity of the polymers increases. As shown in Table S2, when the EGDMA dosage is $50 \mathrm{mg}$, the value of $S C_{\mathrm{P} 4 / \mathrm{E}}$ is maximum. Therefore, $50 \mathrm{mg}$ of EGDMA was selected as the final amount.

\subsection{Characterization of CNT@CS/P(MAA)}

\subsubsection{TEM analysis}

As shown in Fig. 3, the morphology of each material is observed by TEM. Multi-walled carbon nanotubes (Fig. 3A) have a smooth surface and good light transmittance. Compared with CNTs, the light transmittance of CNT@CS (Fig. 3B) is decreased and the surface of CNT@CS is slightly rough, demonstrating that the CS layer has been modified on the surface of the carbon nanotubes. As for CNT@CS/P(MAA) (Fig. 3C), the light transmittance is further decreased, and it is observed that obvious coarse-grained polymers are attached to the surface of the carbon nano-materials, suggesting that the MAA polymers have been grafted onto the surface of CNT@CS.

\subsubsection{FT-IR spectra analysis}

FT-IR spectra of CNTs, CNT@CS and CNT@CS/P(MAA) are shown in Fig. 4. In Fig. 4A, the characteristic peaks of CNTs appearing at 2115, 2016 and $1980 \mathrm{~cm}^{-1}$ are attributed to the stretching vibration of carbon-carbon single and double bonds. In Fig. 4B, the absorbance peaks at 3340, 1389, 1034 and $875 \mathrm{~cm}^{-1}$, correspond to the stretching and bending vibration of $\mathrm{N}-\mathrm{H}, \mathrm{O}-\mathrm{H}$, and $\mathrm{C}-\mathrm{O}-\mathrm{C}$, respectively, indicating that the CS layer is coated on the surface of the CNTs. In Fig. 4C, the peaks at 2980 and $2789 \mathrm{~cm}^{-1}$ (C-H bond stretching vibration), $1465 \mathrm{~cm}^{-1}$ (C-H bond bending vibration), 1106, 1080 and $1030 \mathrm{~cm}^{-1}$ (C-C bond stretching vibration), 1630 and $1389 \mathrm{~cm}^{-1}(\mathrm{C}=\mathrm{O}$ bond stretching vibration) appear, while the peaks at 2115, 2016 and $1980 \mathrm{~cm}^{-1}$ are rapidly decreased, revealing that methacrylate polymers has been wrapped onto the surface of CNTs@CS, which is consistent with the TEM results.

\subsubsection{XRD analysis}

The crystal structure of the CNTs, CNT@CS and CNT@CS/P(MAA) were characterized by XRD. As shown in Fig. S1, the CNT@CS and CNT@CS/P(MAA) exhibit the same XRD signals at (002), (100), (101) and (004) as with CNTs. The diffraction peaks at (002) and (004) are the characteristic diffraction peaks of carbon tubes, which matched well with the database of carbon nanotubes in JCPDS (JCPDS card: 02-0456). The diffraction peak at (100) and (101) was the diffraction peak of high-phase graphite. The CNT, CNT@CS and CNTS@CS/P (MAA) all showed consistent X-ray diffraction peaks, indicating that the crystal structure of the carbon nanotube carriers is not affected by the grafted chitosan layer and methacrylic polymer.

\subsubsection{Nitrogen adsorption-desorption of CNTs and CNT@CS/P(MAA)}

Nitrogen sorption isotherms (Fig. S2) of CNTs and CNT@CS/P(MAA) samples showed similar II isotherm, implying a fast nitrogen gas uptake at relative pressures $<0.10$ and multi-molecular layer adsorption of nitrogen. Table 1 shows that the BET surface area of CNT@CS/P(MAA) sample (87.33 $\left.\mathrm{m}^{2} \cdot \mathrm{g}^{-1}\right)$ is obviously lower than that of CNTs $\left(121.44 \mathrm{~m}^{2} \cdot \mathrm{g}^{-1}\right)$ due to the adhesion of methacrylate polymers layer. While the total pore volume and average pore diameter of the CNT@CS/P(MAA) (0.47 
$\mathrm{cm}^{3} \cdot \mathrm{g}^{-1}$ and $216.82 \AA$, respectively) are higher than these of CNTs $\left(0.40 \mathrm{~cm}^{3} \cdot \mathrm{g}^{-1}\right.$ and $132.50 \AA$, respectively), meaning that CNT@CS/P(MAA) has more mesoporous structures than CNTs. Therefore, CNT@CS/P(MAA) could provide more accessible cavities and binding sites for target analytes, making it easier for the targets to enter the identification cavities.

\subsubsection{Thermogravimetric analysis}

TGA analysis of the CNTs and CNT@CS/P(MAA) were also performed. As shown in Fig. S3, two main weight losses are observed for the two materials. The first weight loss under $100{ }^{\circ} \mathrm{C}$ arise from the volatilization of moisture, where the weight loss of the CNT@CS/P(MAA) is the same as the CNTs. The second weight loss of CNT@CS/P(MAA) over $300{ }^{\circ} \mathrm{C}$ is significantly higher than that of CNTs, especially at $300-500{ }^{\circ} \mathrm{C}$. Unlike bare CNTs, CNT@CS/P(MAA) is coated with a chitosan layer and a methacrylate polymer layer, indicating that the weight loss at $300-500{ }^{\circ} \mathrm{C}$ was a result of chitosan and polymers decomposition. At $500-800^{\circ} \mathrm{C}$, the CNTs and CNT@CS/P(MAA) have the same rate of weight loss due to the decomposition and oxidation of the carbon phase. The results are agreed with the published report (Hua et al. 2018). The CNT@CS/P(MAA) is stable at temperatures up to $300^{\circ} \mathrm{C}$, which indicates its suitability for routine analysis.

\subsection{Adsorption properties}

\subsubsection{Optimization of adsorption conditions}

Adsorption experiments were performed in a methanol-water solution with different water contents, $\mathrm{pH}$ values and temperature. The results are shown in Fig. S4. The adsorption capacity of CNTs@CS/P(MAA) for P4 was increased with the increase of water content (Fig. S4(A)). When the water content increase, the hydrophobic force increase, which drives the analytes into the pores of the material and interacts with the active sites. However, when the water content is too much (over $80 \%$ ), the progesterone crystals would precipitate out at the concentration of $50 \mu \mathrm{g} \cdot \mathrm{mL}^{-1}$. Therefore, methanol: water $(2: 8, \mathrm{~V} / \mathrm{V})$ solution is selected as the adsorption solvent in the subsequent experiments.

Moreover, the influence of the $\mathrm{pH}$ value and the temperature on adsorption capacity were investigated as shown in Fig. S4(B) and Fig. S4(C). When the pH value changed from 3.0 to 11.0, the adsorption capacity of CNT@CS/P(MAA) for P4 did not change significantly, indicating that the adsorption capacity of the prepared materials for $\mathrm{P} 4$ was not affected by the $\mathrm{pH}$ value of aqueous phase. At the same time, the adsorption temperatures had little effect on the adsorption property of the prepared materials. The CNT@CS/P(MAA) has a very stable specific adsorption performance for P4 at temperatures below $45^{\circ} \mathrm{C}$ and different $\mathrm{pH}$ values. Therefore, it could be used for the enrichment of $\mathrm{P} 4$ in the complex industrial wastewater samples.

\subsubsection{Adsorption isotherms}

The adsorption isotherms of CNT@CS/P(MAA) for P4 are shown in Fig. 5. Under different temperatures, the isothermal adsorption curves of CNT@CS/P(MAA) are almost the same. The adsorption capacity of CNT@CS/P(MAA) is significantly increased with increasing initial concentration of $\mathrm{P} 4$, and do not reach equilibrium even though the concentration of $\mathrm{P} 4$ is saturated at $175 \mathrm{mg} \cdot \mathrm{L}^{-1}$. At the concentration of $175 \mathrm{mg} \cdot \mathrm{L}^{-1}$, the maximum adsorption capacity of CNT@CS/P(MAA) to P4 is 44.45 $\mathrm{mg} \cdot \mathrm{g}^{-1}$, which is higher than that reported in the literatures (Hao et al. 2015, Li et al. 2020, Zheng et al. 2018). To further verify the binding properties of CNT@CS/P(MAA), the Langmuir model and 
Freundlich model were applied to fit the data, and relevant parameters were calculated. Two equations are expressed by Eq. (5) and Eq. (6), respectively.

$$
\begin{aligned}
& C_{\mathrm{e}} / Q_{\mathrm{e}}=C_{\mathrm{e}} / Q_{\mathrm{m}}+1 / Q_{\mathrm{m}} \times K_{1} \\
& \ln Q_{e}=\ln C_{\mathrm{e}} / \mathrm{n}+\ln K_{\mathrm{f}}
\end{aligned}
$$

where $Q_{\mathrm{e}}\left(\mathrm{mg} \cdot \mathrm{g}^{-1}\right)$ is the adsorption capacity at equilibrium; $C_{\mathrm{e}}\left(\mathrm{mg} \cdot \mathrm{L}^{-1}\right)$ is the equilibrium concentration of P4; $K_{1}$ and $Q_{\mathrm{m}}$ are the Langmuir constant and the maximum theoretical adsorption capacity, respectively; $K_{\mathrm{f}}$ and $\mathrm{n}$ are the Freundlich constant and heterogeneity factor, respectively.

As shown in Table 2, the correlation coefficients of Freundlich model were higher $\left(R^{2} \geq 0.9645\right)$ than those of Langmuir model $\left(\mathrm{R}^{2} \leq 0.9095\right)$ under different temperatures. This signified that the Freundlich model could more accurately described the isotherm data of CNT@CS/P(MAA). The Freundlich isotherm model assumes a multilayer adsorption occurring on a heterogeneous surface, and the heat of adsorption is not uniform between the molecules adsorbed onto the surface of adsorbent. Therefore, the adsorption process of CNT@CS/P(MAA) for P4 was multilayer adsorption behavior, or the adsorption of P4 occurs on a heterogeneous interface between the solution and CNT@CS/P(MAA).

\subsubsection{Adsorption kinetics}

Fig. 6 shows the kinetic curve of CNT@CS/P(MAA) for P4 at $25{ }^{\circ} \mathrm{C}$. It is obvious that the adsorption capacity increases rapidly in the first few minutes and reaches the adsorption equilibrium within 60 minutes. The saturation adsorption capacity of CNT@CS/P(MAA) is $18.98 \mathrm{mg} \cdot \mathrm{g}^{-1}$ at the concentration of $50 \mu \mathrm{g} \cdot \mathrm{mL}^{-1}$. Then the pseudo-first-order rate Eq. (7) and pseudo-second-order rate Eq. (8) are used to fit the kinetic data, and the results are showed in Table 3.

$$
\begin{aligned}
& \lg Q_{\mathrm{e}} / Q_{\mathrm{t}}=\lg Q_{\mathrm{e}}-k_{1} \times \mathrm{t} / 2.303 \\
& \mathrm{t} / Q_{\mathrm{t}}=1 /\left(k_{2} \times Q_{\mathrm{e}}{ }^{2}\right)+\mathrm{t} / Q_{\mathrm{e}}
\end{aligned}
$$

where $k_{1}$ and $k_{2}$ are the adsorption rate constants of the pseudo-first-order equation and pseudo-secondorder equation, respectively; $Q_{\mathrm{e}}\left(\mathrm{mg} \cdot \mathrm{g}^{-1}\right)$ is the adsorption capacity at equilibrium, and $Q_{\mathrm{t}}\left(\mathrm{mg} \cdot \mathrm{g}^{-1}\right)$ is the adsorption capacity at time $t$.

As shown in Table 3, compared with the pseudo-first-order kinetic model, the regression correlation coefficient of the pseudo-second-order kinetic model $\left(\mathrm{R}^{2}=0.9745\right)$ is higher. The theoretical maximum adsorption capacity $Q_{\mathrm{e}}$ calculated by the pseudo-second-order model $\left(18.60 \mathrm{mg} \cdot \mathrm{g}^{-1}\right)$ is closer to the experimental value $\left(18.98 \mathrm{mg} \cdot \mathrm{g}^{-1}\right)$. The results demonstrated that the adsorption of $\mathrm{P} 4$ onto CNT@CS/P(MAA) followed the pseudo-second-order kinetic model. Hence, the adsorption rate is limited by chemisorption which involved the electron sharing or transfer between CNT@CS/P(MAA) and P4.

\subsubsection{Adsorption selectivity}

E, E2, DXM, DES and BPA (Fig. 1) were chosen to study the selectivity of the prepared polymers. The selection coefficient is a quantitative parameter used to evaluate the discrimination ability of different materials for the target analyte from interfering analogues. The $S C$ was calculated by Eq. (4) and the results are shown in Table 4. Compared with CNTs and CNT@CS, the selectivity of CNT@CS/P(MAA) for P4 is significantly improved. The calculated $S C$ values for E, E2, DXM, DES and BPA are 2.42, 2.46, 10.03, 18.94, and 34.43, respectively, indicating the high specificity of CNT@CS/P(MAA) towards P4. There are differences between P4 and the five analogues in the steroidal 
ring, three-dimensional structure and functional group, which lead to their different spatial distribution and different interactions with the active sites in the polymer cavities, resulting in a high selectivity for P4.

The plentiful carboxyl in MAA polymers provided adsorption sites for binding to carbonyl and hydroxyl groups. Therefore, the adsorption capacity of CNT@CS/P(MAA) mainly depends on the amount of carboxyl groups and the pore size of the materials surface. P4, E, E2 and DXM contain carbonyl or hydroxyl groups, which could interact more favorably with the carboxyl in MAA polymers. Among them, E and E2 have a spatial structure similar to that of P4, and could more freely enter the holes of the MAA polymers. Moreover, both the C3-carbonyl and C17-methyl ketones of P4 can interact electrostatically with the carboxyl groups of the MAA polymers, while E and E2 only have C17-carbonyl or hydroxyl that could interact with the polymers' carboxyl. The force of $\mathrm{E}$ and $\mathrm{E} 2$ is weaker than that of P4. DXM is a cortisol hormone. Although it contains two carbonyl groups and three hydroxyl groups, DXM differs greatly from $\mathrm{P} 4$ in spatial structure and could not easily enter the MAA polymers. Therefore, the selection coefficient of DXM is higher than that of E and E2. DES and BPA are similar to estrogen in pharmacological action, but their spatial structures and action sites are very different from $\mathrm{P} 4$, so the selection coefficients are higher than others.

\subsection{Optimization of SPE conditions}

To selectively enrich P4 and remove impurities from water samples, an acid-functionalized adsorbent was used for SPE, and acetic acid or ammonia were used during the elution to promote the desorption of P4. The recoveries of P4 under different conditions are shown in Fig 7. The highest recovery was obtained when the loading volume, elution solvent, and elution volume were $30 \mathrm{~mL}$, methanol/acetic acid $(2: 1, \mathrm{v} / \mathrm{v})$, and $3 \mathrm{~mL}$, respectively. As shown in Fig.7A, the recovery slightly declined when the loading volume of $\mathrm{P} 4$ solution exceed $30 \mathrm{~mL}$, because the excessive loading volume will cause the oversaturation of binding sites. Therefore, a loading volume of $30 \mathrm{~mL}$ was selected for subsequent experiments. The type of eluent also had a great influence on the recovery of $\mathrm{P} 4$, as shown in Fig.7B, the recovery of $\mathrm{P} 4$ reach a maximum of $95.3 \%$ when the eluent is chloroform/acetic acid (9:1, $\mathrm{v} / \mathrm{v}$ ). Considering the toxicity of chloroform and the requirements of environmental protection, methanol/acetic acid (2:1, v/v) with the second highest recovery $(82.2 \%)$ was chosen as the optimized eluent. Moreover, the elution volume would affect the recovery of P4. When the volume of eluent is too small, the target molecules cannot be completely eluted; while the eluent efficiency of impurities will be increased if the volume of eluent is large enough. As showed in Fig. 7C, when the eluting solution volume was $3 \mathrm{~mL}$, the recovery of $\mathrm{P} 4$ reach the maximum (96.7\%). Therefore, $3 \mathrm{~mL}$ of the methanol/acetic acid $(2: 1, \mathrm{v} / \mathrm{v})$ was selected as the final elution solvent.

\subsection{Method validation and application to industrial wastewater samples}

The SPE-HPLC method was validated for P4 in tap-water. Fig. 8 shows the chromatograms of P4 standard samples and spiked tap-water samples before and after SPE treatment. It showed that the coexisting impurities in the tap-water samples without SPE pretreatment could interfere with the detection of P4. Obviously, the peak areas of the P4 were significantly enhanced after the SPE pretreatment, and the interference of the impurities were effectively reduced. These results suggest that the established SPE-HPLC method could effectively eliminate the influence of co-existing impurities in water and enrich 
the target molecule-P4 in water samples. The linearity of the established analytical method was examined from calibration curves using a series of spiked samples with concentrations ranging from 0.1 to 600 $\mu \mathrm{g} \cdot \mathrm{L}^{-1}$. The correlation coefficient of the regression curve was 0.9998 , showing a good linearity relationship.

The limit of detection (LOD) of the method was evaluated with a signal-to noise ratio of three (S/N $=3$ ), and the limit of quantification $(\mathrm{LOQ})$ was determined with a signal-to-noise ratio of ten $(\mathrm{S} / \mathrm{N}=10)$. And the LOD and LOQ were $3 \mathrm{ng} \cdot \mathrm{L}^{-1}$ and $10 \mathrm{ng} \cdot \mathrm{L}^{-1}$, respectively. To test the reusability of the proposed method, the same SPE column was used to continuously extract P4 in the aqueous phase. The results (Figure S5) show that the recovery was $\geq 96.3 \%$ after recycling 5 times, indicating that the method had a good reusability.

The accuracy and precision of this method were evaluated by determining the repeatability and reproducibility of the established method (Table 5). The repeatability and reproducibility were measured over a five-day period and three SPE columns were used under the same conditions. The accuracy of this method is ranged from $96.3-104.2 \%$, and the RSD values are less than $5.6 \%$, indicating that the established SPE-HPLC method has a good accuracy and precision. Therefore, this method could meet the detection requirements of trace progesterone in the environmental water samples.

Then, we detected the dosage of P4 in real industrial wastewater samples using the established method. In the No. 1 samples collected near the industrial production, the concentration of P4 detected after SPE pretreatment was $0.48 \mathrm{mg} \cdot \mathrm{L}^{-1}$, which was 1.78 times the $\mathrm{P} 4$ concentration $(0.27 \mathrm{mg} \cdot \mathrm{L}-1)$ without SPE treatment. Meanwhile, in the No. 2 samples collected at the end of the sewage treatment system, $0.003 \mathrm{mg} \cdot \mathrm{L}^{-1}$ of $\mathrm{P} 4$ was detected by the established SPE-HPLC method, while no progesterone was detected by HPLC without SPE treatment. These results show that even in complex industrial production wastewater samples, the established SPE-HPLC method could still effectively enrich P4 and achieve the detection of $\mathrm{P} 4$ at a very low concentration. Therefore, the proposed method is suitable for the effective enrichment and detection of $\mathrm{P} 4$ in the industrial wastewater.

\subsection{Comparison study}

The developed SPE-HPLC method was compared with previously reported methods(Hao et al. 2015, Li et al. 2020, Lucci et al. 2011, Mirzajani et al. 2019b, Razmkhah et al. 2018, Zhang et al. 2020a, Zheng et al. 2018). The results are listed in Table 6. Compared with other methods, the developed SPE-HPLC method exhibited excellent adsorption capacity, outstanding sensitivity and relatively low detection limits. This method also provided a new strategy for the detection of steroid hormones in the wastewater and environmental samples.

\section{Conclusions}

In this study, CNT@CS/P(MAA) were fabricated by using a simple procedure, and served as novel extraction sorbents for the specific enrichment of P4 in the industrial wastewater samples. The results demonstrated that the fabricated carbon materials exhibited excellent adsorption capacity, high selectivity and prominent sensitivity, and was almost unaffected by the ambient temperature and $\mathrm{pH}$ value. The high selectivity of CNT @ CS/P(MAA) for P4 was mainly due to the steric effect and hydrogen bonding force between them. Additionally, the method validation showed a good linearity, satisfactory recoveries and stable repeatability. The established method could effectively enrich and detect P4 in the 
actual industrial wastewater samples without the interference of matrix complexity. Compared with the published reports, the proposed method has the characteristics of large adsorption capacity, stable adsorption performance and high sensitivity. It could be a promising alternative for P4 monitoring in the industrial wastewater, and provides a new method for the detection of $\mathrm{P} 4$ in other environmental waters.

\section{Declarations:}

\section{Ethics approval and consent to participate}

Not applicable

\section{Consent for publication}

Not applicable

\section{Availability of data and materials}

All data generated or analysed during this study are included in this published article and its supplementary information files.

\section{Competing interests}

The authors declare that they have no known competing financial interests or personal relationships that could have appeared to influence the work reported in this paper.

\section{Funding}

This study was financially supported by the National Natural Science Foundation of China (No. 81773689).

\section{Authors' contributions}

Xia Cui (cuixia0415@ stu.xjtu.edu.cn): Conceptualization, methodology, formal analysis, investigation, visualization, writing — original draft

Hua Shu (shuhuas@stu.xjtu.edu.cn): Methodology, formal analysis, writing—review and editing

Lu Wang (wanglu0914@mail.xjtu.edu.cn):Writing—review and editing

Guoning Chen (chengn1992@stu.xjtu.edu.cn):Writing_review and editing

Jili Han (HJL1995061968@stu.xjtu.edu.cn): Writing—review and editing

Qianqian Hu (huqiangqian@ stu.xjtu.edu.cn): Writing—review and editing

Kamran Bashir (kamran2016@stu.xjtu.edu.cn): Writing_review and editing

Zhimin Luo (luozm0905@xjtu.edu.cn): Visualization,writing_review and editing

Chun Chang (changchun@mail.xjtu.edu.cn): Supervision, funding acquisition

Jia Zhang (zadam@sxhjp.com): Funding acquisition

Qiang Fu (fuqiang@mail.xjtu.edu.cn): Conceptualization, visualization, supervision, funding acquisition

\section{Acknowledgements}

The authors thank the National Natural Science Foundation of China for providing financial support and the Instrumental Analysis Center of Xi'an Jiaotong University for providing excellent characterization platform.

\section{Authors' information}

\section{Affiliations}


Xia Cui, Hua Shu, Lu Wang, Guoning Chen, Jili Han, Qianqian Hu, Kamran Bashir, Zhimin Luo, Chun

Chang, Qiang Fu.

Institute of Drug Safety and Monitoring, Academy of Pharmaceutical Science and Technology,

Xia Cui, Hua Shu, Lu Wang, Guoning Chen, Jili Han, Qianqian Hu, Kamran Bashir, Zhimin Luo, Chun

Chang, Qiang Fu.

\section{Shaanxi Hanjiang Pharmaceutical Group Co., Ltd, Hanzhong 723000, China}

Jia Zhang, Qiang Fu.

\section{Reference:}

Akshaya KB, Bhat VS, Varghese A, George L, Hegde G (2019): Non-Enzymatic Electrochemical Determination of Progesterone Using Carbon Nanospheres from Onion Peels Coated on Carbon Fiber Paper. J. Electrochem. Soc. 166, B1097-B1106

Chamas A, Pham HTM, Jahne M, Hettwer K, Gehrmann L, Tuerk J, Uhlig S, Simon K, Baronian K, Kunze G (2017): Separation and identification of hormone-active compounds using a combination of chromatographic separation and yeast-based reporter assay. Sci. Total Environ. 605-606, 507-513

Chen J, Huang Y, Liang C, Wang B (2018): Preparation and electromagnetic shielding performance of functionalized carbon nanotube / epoxy porous composites. Chem. Ind. \& Eng. Pro.(China) 37, 664-672

De Lima CA, Spinelli A (2013): Electrochemical behavior of progesterone at an ex situ bismuth film electrode. Electrochim. Acta 107, 542-548

Du W, Sun M, Guo P, Chang C, Fu Q (2018): Molecularly imprinted membrane extraction combined with high-performance liquid chromatography for selective analysis of cloxacillin from shrimp samples. Food Chem. 259, 73-80

Fabbrocini A, Coccia E, D'Adamo R, Faggio C, Paolucci M (2019): Mifepristone affects fertility and development in the sea urchin Paracentrotus lividus. Mol. Reprod. Dev. 86, 1348-1356

Graham JD, Clarke CL (1997): Physiological action of progesterone in target tissues. Endocr. Rev. 18, 502-19

Guo P, Chen G, Shu H, Li P, Yu P, Chang C, Wang Y, Fu Q (2019): Monodisperse molecularly imprinted microsphere cartridges coupled with HPLC for selective analysis of dexamethasone and hydrocortisone in cosmetics by using matrix solid-phase dispersion. Anal. Methods 11, 36873696

Guo R, Li L, Wang Y, Wang L, Zhang M, Zhan W, Shi Q (2016): Historical story on natural medicinal chemistry: Steroids. Chin. Tradit. Herb Drugs 47, 1251-1264

Guohua H, Hongyang L, Zhiming J, Danhua Z, Haifang W (2017): Study of small-cell lung cancer cellbased sensor and its applications in chemotherapy effects rapid evaluation for anticancer drugs. Biosens. Bioelectron. 97, 184-195

Hao Y, Gao R, Shi L, Liu D, Tang Y, Guo Z (2015): Water-compatible magnetic imprinted nanoparticles served as solid-phase extraction sorbents for selective determination of trace 17beta-estradiol in environmental water samples by liquid chromatography. J. Chromatogr. A 1396, 7-16

Hashmi MAK, Escher BI, Krauss M, Teodorovic I, Brack W (2018): Effect-directed analysis (EDA) of Danube River water sample receiving untreated municipal wastewater from Novi Sad, Serbia. Sci. Total Environ. 624, 1072-1081

Hashmi MAK, Krauss M, Escher BI, Teodorovic I, Brack WJeoTTE (2020): Effect-Directed Analysis of Progestogens andGlucocorticoids at Trace Concentrations in River Water. Environ. Toxicol. Chem. 39, 189-199

Hua S, Yanhui G, Xin-Ya X, Peng-Qi G, Zhi-Min L, Wei D, Chun C, Rui-Lin L, Qiang FJJoCA (2018): Hybrid-type carbon microcoil-chitosan composite for selective extraction of aristolochic acid I from Aristolochiaceae medicinal plants. J. Chromatogr. A 1561, 13-19

Jakubus A, Paszkiewicz M, Stepnowski P (2016): Carbon Nanotubes Application in the Extraction Techniques of Pesticides: A Review. Anal. Chem. 47, 76-91 
Khan MS, Dighe K, Wang Z, Srivastava I, Schwartz-Duval AS, Misra SK, Pan D (2019): Electrochemical-digital immunosensor with enhanced sensitivity for detecting human salivary glucocorticoid hormone. Analyst 144, 1448-1457

Kollofrath D, Geppert M, Polarz S (2020): Copolymerization of Mesoporous Styrene-Bridged Organosilica Nanoparticles with Functional Monomers for the Stimuli-Responsive Remediation of Water. Chemsuschem. 13, 5100-5111

Kumar V, Johnson AC, Trubiroha A, Tumova J, Ihara M, Grabic R, Kloas W, Tanaka H, Kroupova HK (2015): The challenge presented by progestins in ecotoxicological research: a critical review. Environ. Sci. Technol. 49, 2625-38

Li L, Chen Y, Yang Y, Yang Y, Yang L, Wang Z (2020): Rapid and sensitive analysis of progesterone by solid-phase extraction with amino-functionalized metal-organic frameworks coupled to direct analysis in real-time mass spectrometry. Anal. Bioanal. Chem. 412, 2939-2947

Liu D, Liu C, Tang Q (2018): Thermoelectric effect and mechanical properties of multi-walled carbon nanotubes / ethylene propylene diene monomer composites. New Chem. Mater. 46, 129-132

Liu R, Mao S, Wang Y, Wang L, Ge Y, Xu X, Fu Q (2017): A mussel-inspired hybrid copolymer adhered to chitosan-coated micro-sized carbon fiber aerogels for highly efficient nanoparticle scavenging. Environ. Sci.: Nano 4, 2164-2174

Lucci P, Nunez O, Galceran MT (2011): Solid-phase extraction using molecularly imprinted polymer for selective extraction of natural and synthetic estrogens from aqueous samples. J. Chromatogr. A 1218, 4828-4833

Luque-Cordoba D, Lopez-Bascon MA, Priego-Capote F (2020): Development of a quantitative method for determination of steroids in human plasma by gas chromatography-negative chemical ionization-tandem mass spectrometry. Talanta 220, 121415

Mirzajani R, Kardani F, Ramezani Z (2019a): A nanocomposite consisting of graphene oxide, zeolite imidazolate framework 8, and a molecularly imprinted polymer for (multiple) fiber solid phase microextraction of sterol and steroid hormones prior to their quantitation by HPLC. Microchim. Acta 186, 129-143

Mirzajani R, Kardani F, Ramezani Z (2019b): A nanocomposite consisting of graphene oxide, zeolite imidazolate framework 8, and a molecularly imprinted polymer for (multiple) fiber solid phase microextraction of sterol and steroid hormones prior to their quantitation by HPLC. Microchim. Acta 186

Nezhadali A, Es'haghi Z, Khatibi A (2016): Selective extraction of progesterone hormones from environmental and biological samples using a polypyrrole molecularly imprinted polymer and determination by gas chromatography. Anal. Methods 8, 1813-1827

Razmkhah K, Sereshti H, Soltani S, Nodeh HR (2018): Extraction and Determination of Three Steroid Molecules in Milk Using Functionalized Magnetic Carbon Nanotube-Based Solid Phase Extraction Coupled with HPLC. Anal. Methods 11, 3179-3189

Shu H, Chen G, Wang L, Cui X, Wang Q, Li W, Chang C, Guo Q, Luo Z, Fu Q (2020): Adenine-coated magnetic multiwalled carbon nanotubes for the selective extraction of aristolochic acids based on multiple interactions. J. Chromatogr. A 1627, 461382

Siren H, El Fellah S (2017): Androgens, oestrogens, and progesterone concentrations in wastewater purification processes measured with capillary electrophoresis. Environ. Sci. Pollut. Res. 24, 16765-16785

Tao X, Wang X, Liu B, Liu J (2020): Conjugation of antibodies and aptamers on nanozymes for developing biosensors. Biosens. Bioelectron. 168, 112537

Tian X, Song H, Wang Y, Tian X, Tang Y, Gao R, Zhang C (2020): Hydrophilic magnetic molecularly imprinted nanobeads for efficient enrichment and high performance liquid chromatographic detection of 17beta-estradiol in environmental water samples. Talanta 220, 121367

Xu W, Jiao L, Ye H, Guo Z, Wu Y, Yan H, Gu W, Du D, Lin Y, Zhu C (2020): pH-responsive allochroic nanoparticles for the multicolor detection of breast cancer biomarkers. Biosens. Bioelectron. 148,111780

Xu X, Zheng QQ, Bai GM, Dai QM, Cao XD, Yao YW, Liu SQ, Yao C (2018): Polydopamine functionalized nanoporous graphene foam as nanoreactor for efficient electrode-driven metabolism of steroid hormones. Biosens. Bioelectron. 119, 182-190

Xu XY, Guo PQ, Luo ZM, Ge YH, Zhou YL, Chang RM, Du W, Chang C, Fu Q (2017): Preparation and characterization of surface molecularly imprinted films coated on multiwall carbon nanotubes for recognition and separation of lysozyme with high binding capacity and selectivity. RSC Adv. 7, 18765-18774 
Yu Q, Geng J, Zong X, Zhang Y, Xu K, Hu H, Deng Y, Zhao F, Ren H (2019): Occurrence and removal of progestagens in municipal wastewater treatment plants from different regions in China. Sci. Total Environ. 668, 1191-1199

Zhang J, Zang L, Wang T, Wang X, Jia M, Zhang D, Zhang H (2020a): A solid-phase extraction method for estrogenic disrupting compounds based on the estrogen response element. Food Chem. 333, 127529

Zhang J, Zang L, Wang T, Wang X, Zhang HJFC (2020b): A solid-phase extraction method for estrogenic disrupting compounds based on the estrogen response element. Food Chem. 333, 127529

Zhang K, Zhao Y, Fent K (2017): Occurrence and Ecotoxicological Effects of Free, Conjugated, and Halogenated Steroids Including 17alpha-Hydroxypregnanolone and Pregnanediol in Swiss Wastewater and Surface Water. Environ. Sci. Technol. 51, 6498-6506

Zhang Z, Kang X, Guo Y, Zhang J, Xie J, Shao S, Xiang Y, Chen G, Yu X (2020c): Association of circulating galectin-3 with gestational diabetes mellitus, progesterone, and insulin resistance. J. Diabetes, 1-9

Zheng P, Zhang B, Luo Z, Du W, Guo P, Zhou Y, Chang R, Chang C, Fu Q (2018): Facile preparation of polydopamine-coated imprinted polymers on the surface of $\mathrm{SiO} 2$ for estrone capture in milk samples. J. Sep. Sci. 41, 2585-2594

Zong Y, Chen J, Hou J, Deng W, Liao X, Xiao Y (2018): Hexafluoroisopropanol-alkyl carboxylic acid high-density supramolecular solvent based dispersive liquid-liquid microextraction of steroid sex hormones in human urine. J. Chromatogr. A 1580, 12-21 
Figures
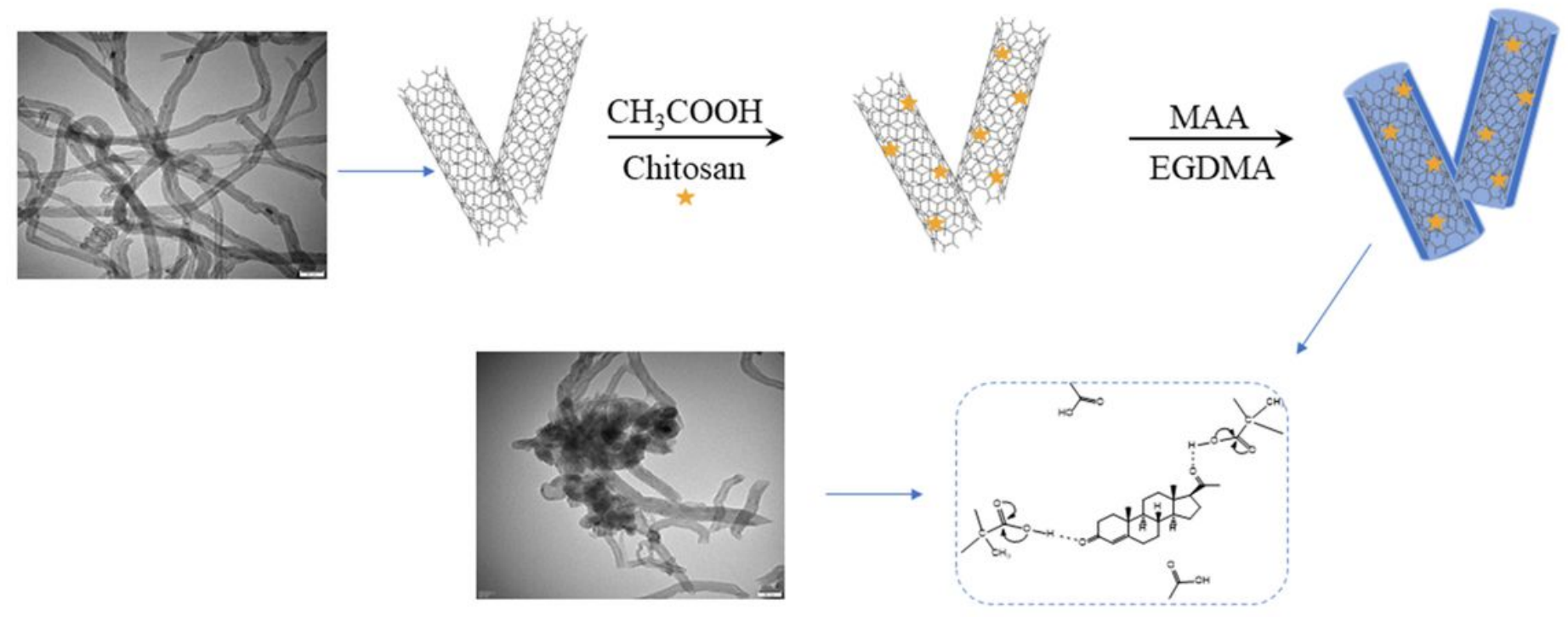

Carbon nanotubes

Chitosan

Poly(methacrylic acid) layer

\section{Figure 1}

The chemical structure of progesterone, estrone, estradiol, dexamethasone, diethylstilbestrol and bisphenol A. 


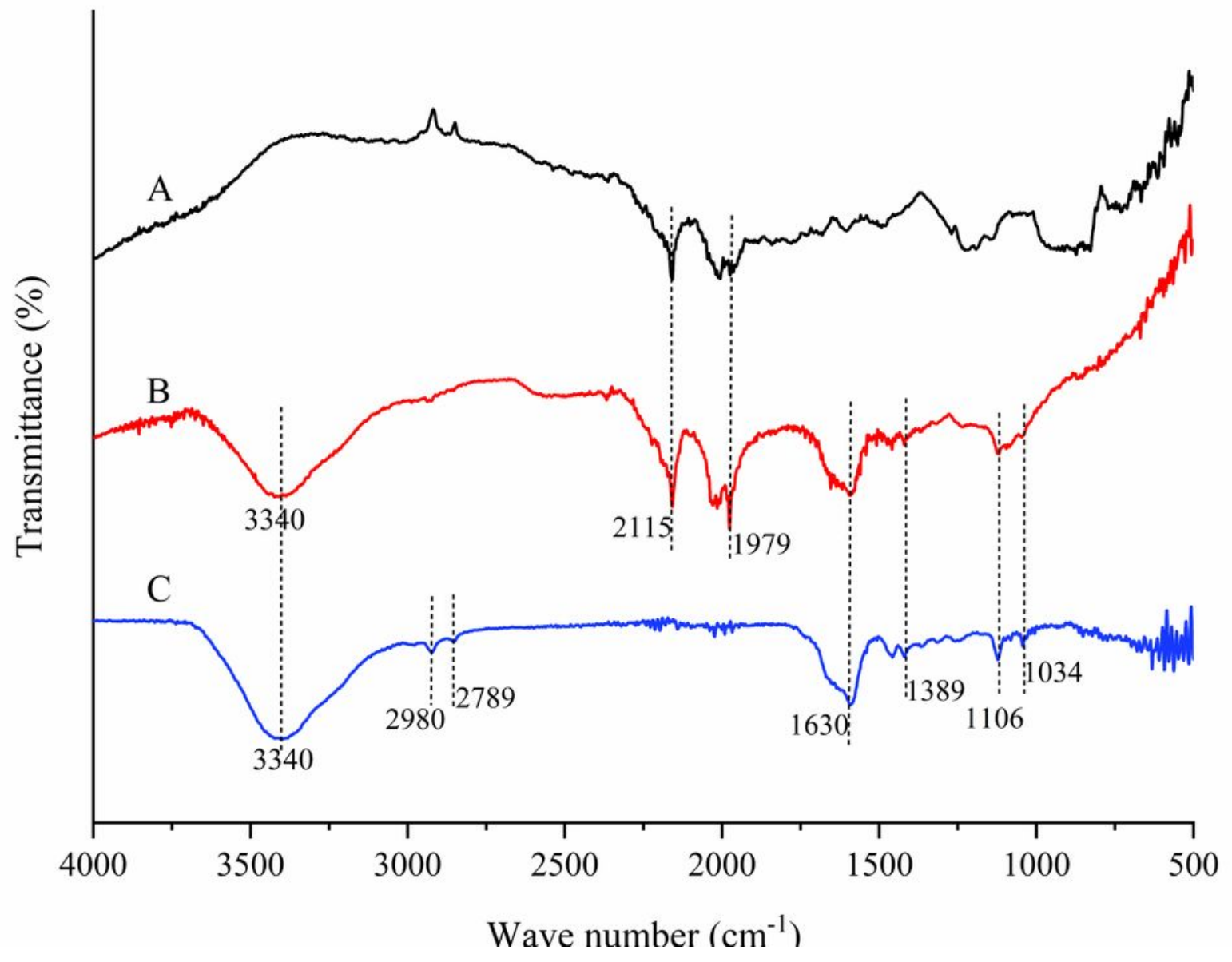

Figure 2

Schematic illustration of the synthesis procedure of the CNT@CS/P(MAA).
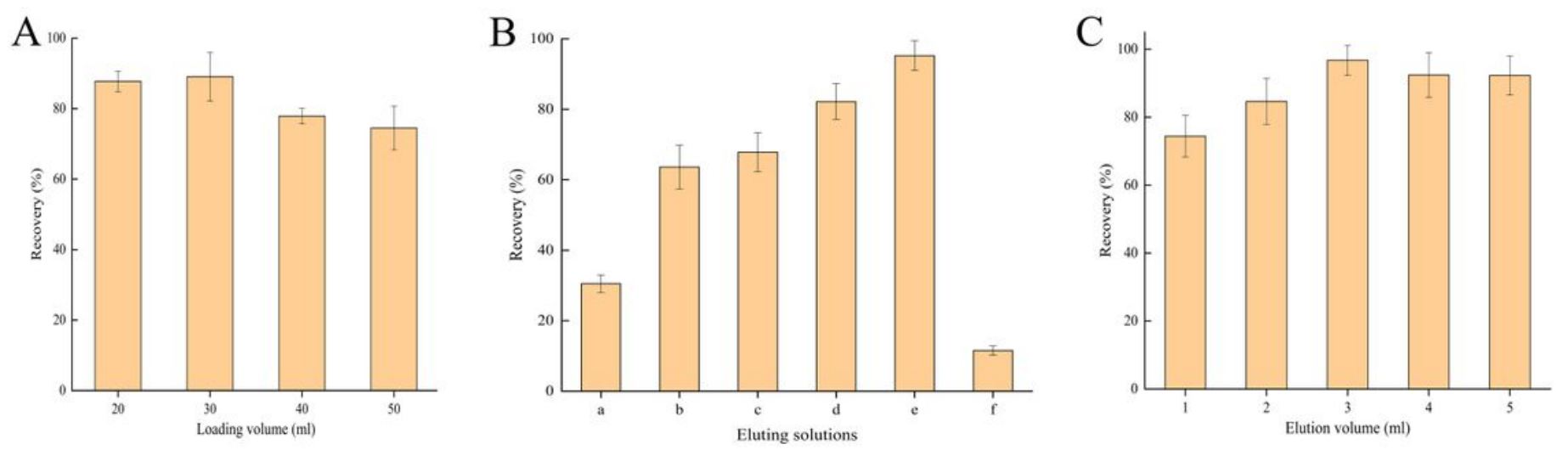

Figure 3

TEM images of the bare CNTs (A), CNT@CS (B), CNT@CS/P(MAA) (C). 


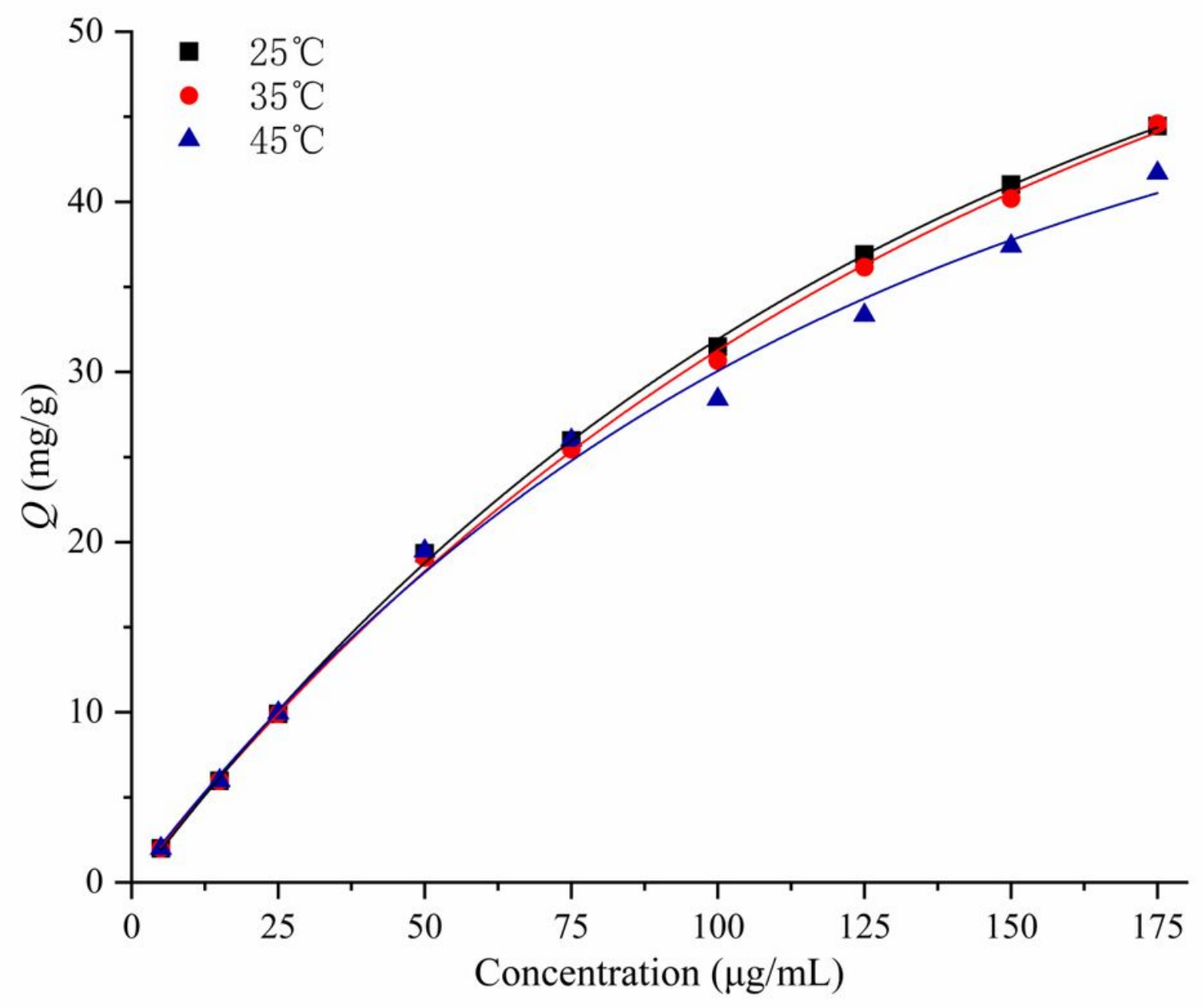

Figure 4

FT-IR spectra of the bare CNTs (A), CNT@CS (B), CNT@CS/P(MAA) (C). 


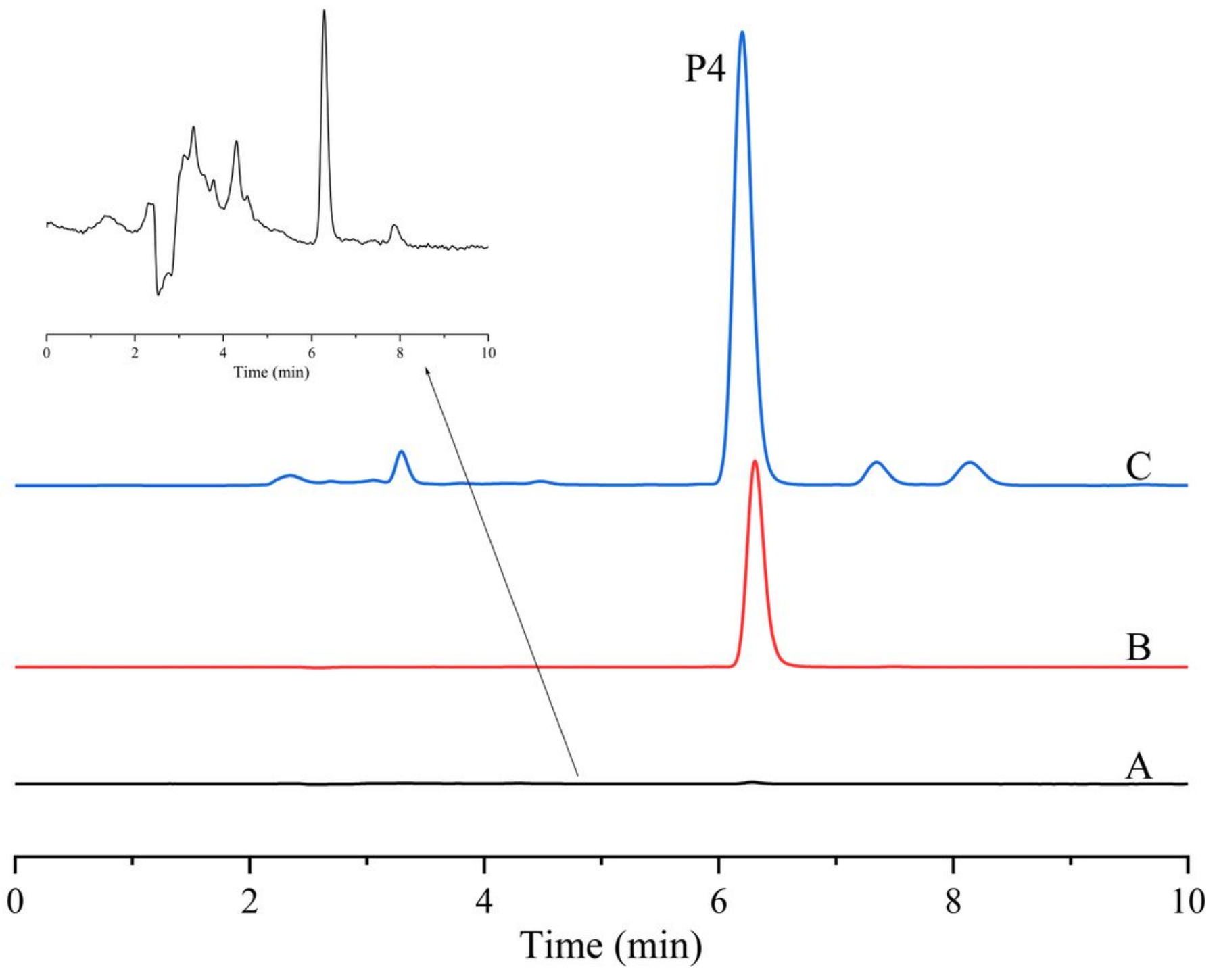

Figure 5

The adsorption isotherms of CNT@CS/P(MAA) at 25区, 35囚 and 45区. 


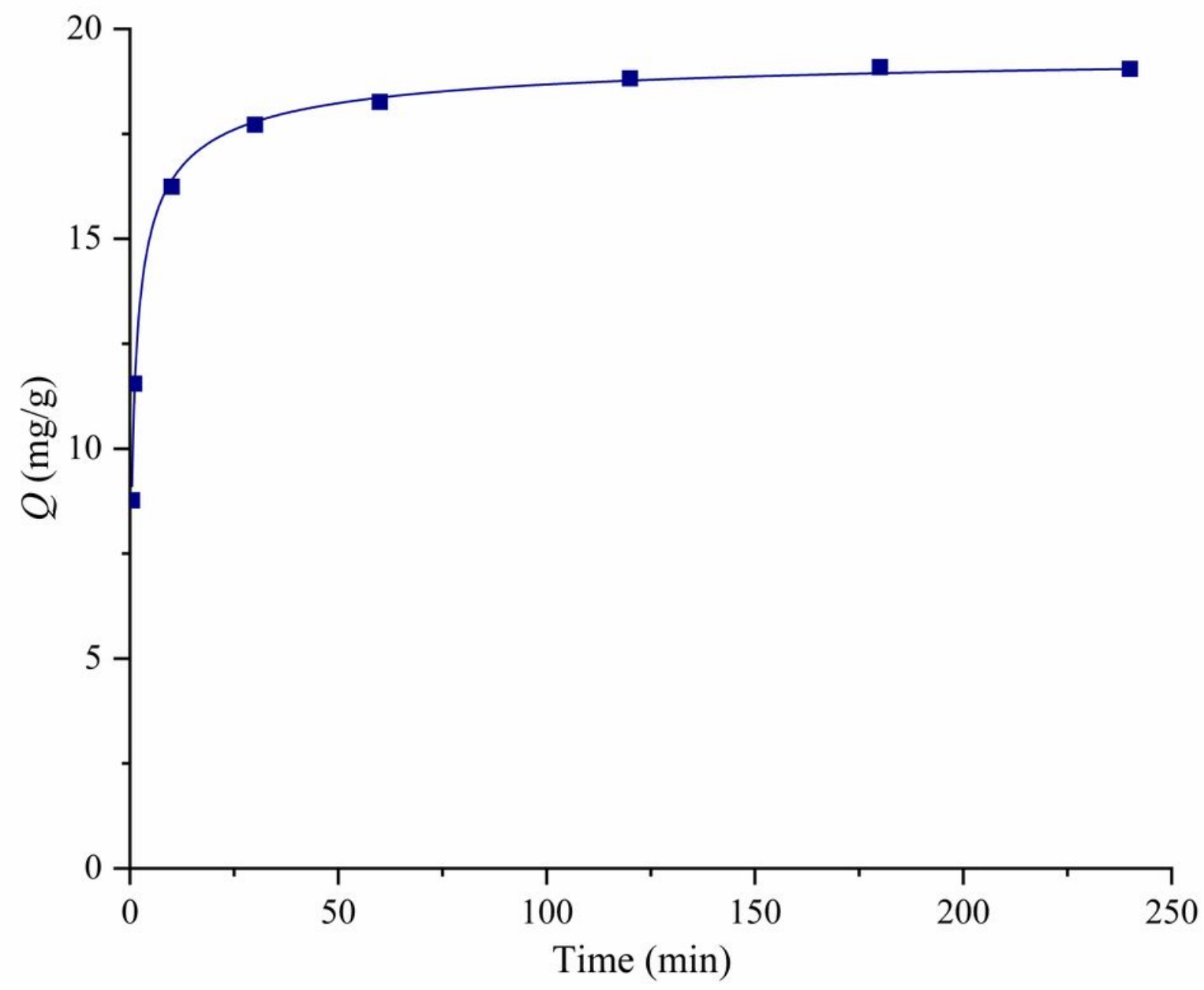

Figure 6

The adsorption kinetics of CNT@CS/P(MAA) under ambient temperature. 


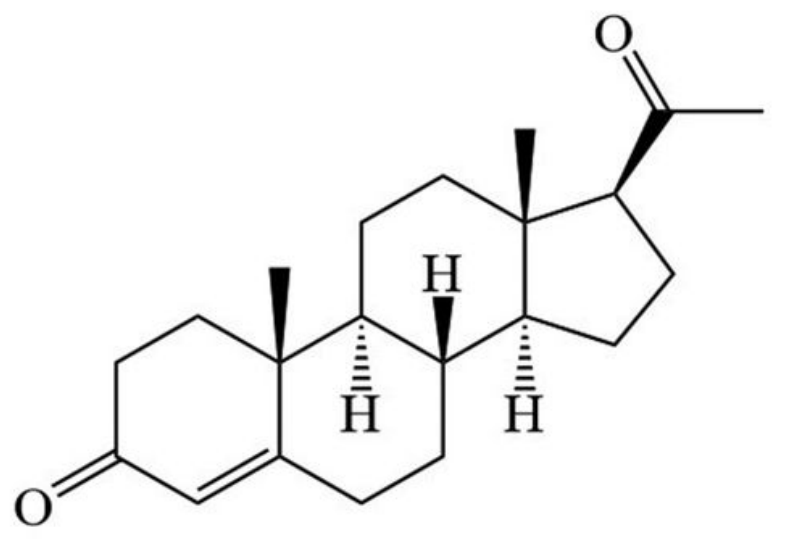

P4

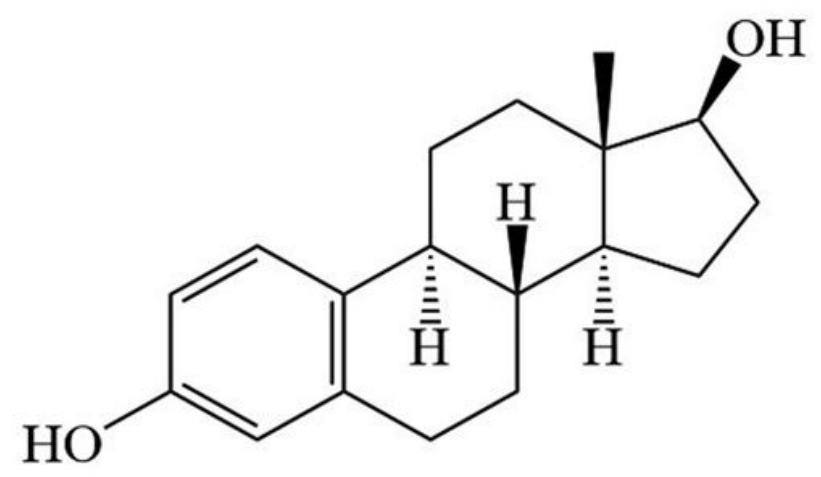

E2

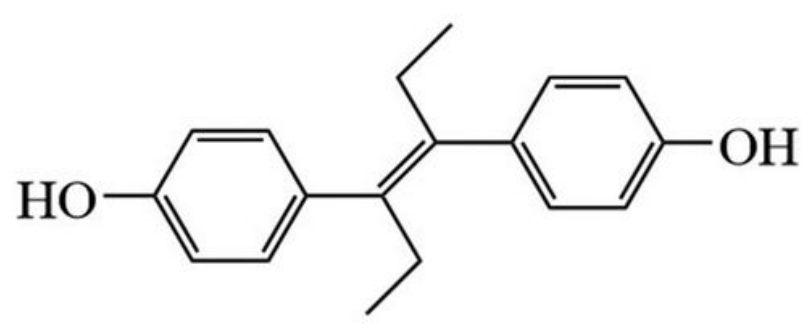

DES

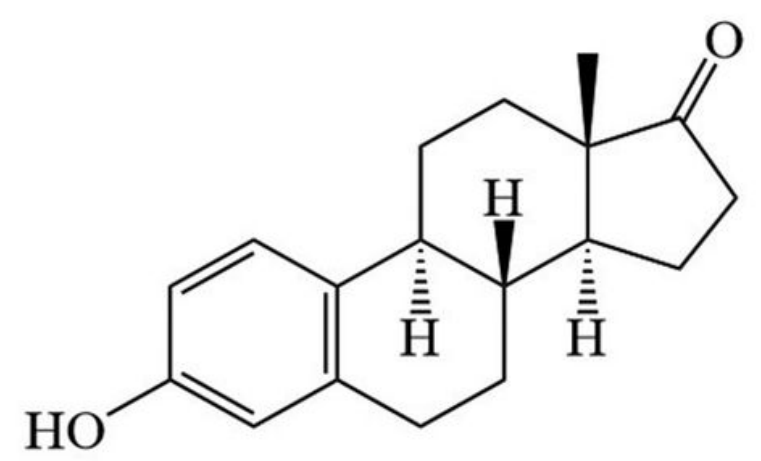

$\mathrm{E}$

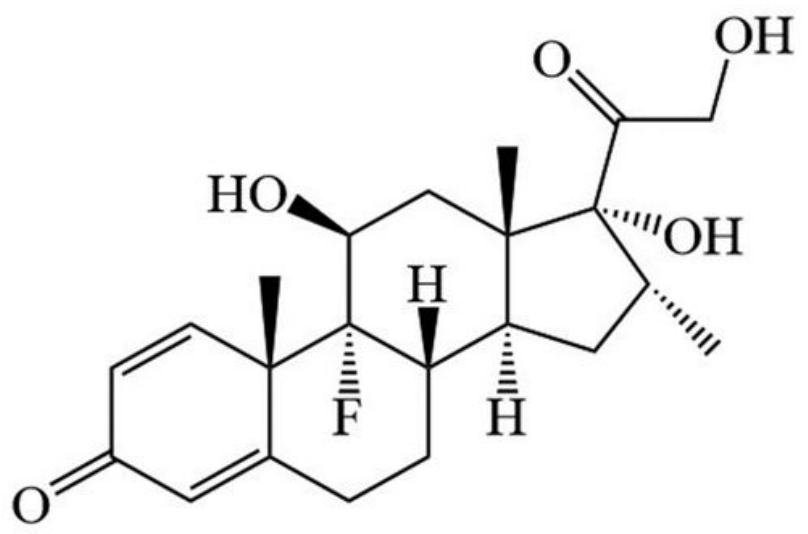

DXM

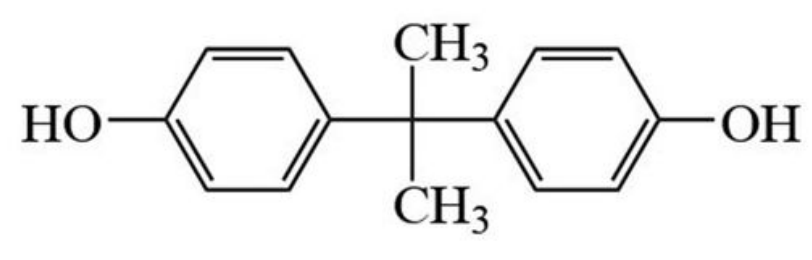

BPA

Figure 7

Optimization results of SPE conditions. A: effect of loading volume on the recoveries of P4; B: effect of eluting solutions on the recoveries of P4 (a: methanol b: methanol-acetic acid (9:1, v/v) c: methanolacetic acid $(4: 1, v / v)$ d: methanol-acetic acid $(2: 1, v / v)$ e: chloroform-acetic acid $(9: 1, v / v)$ f: methanol-25\% ammonia $(9: 1, \mathrm{v} / \mathrm{v}))$; C: effect of elution volume on the recoveries of P4. 


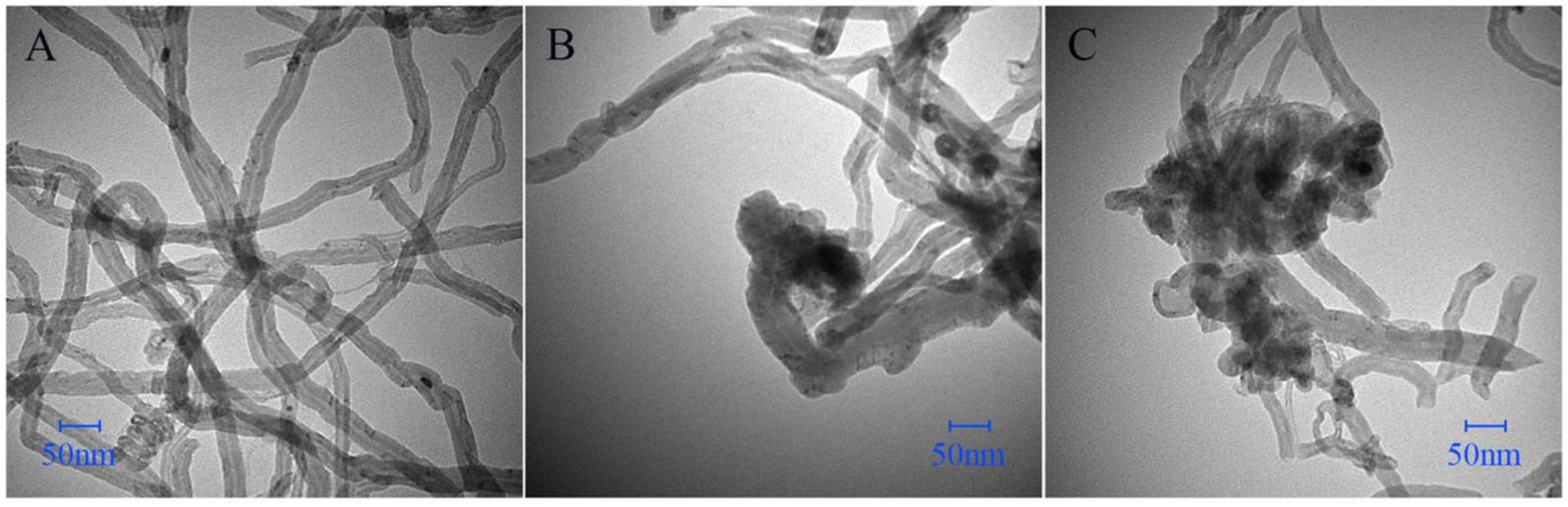

Figure 8

The chromatograms of P4 in tap-water samples before and after treated with SPE. (A) sample before treated with SPE; (B) standard solution of P4; (C) sample after treated with SPE.

\section{Supplementary Files}

This is a list of supplementary files associated with this preprint. Click to download.

- Supplementarymaterial.docx 